\title{
CERTAIN CLASSES OF SOLUTIONS OF LAGERSTROM EQUATIONS
}

\author{
ALMA OMERSPAHIĆ AND VAHIDIN HADŽIABDIĆ
}

\begin{abstract}
This paper presents sufficient conditions for the existence of solutions for certain classes of Cauchy's solutions of the Lagerstrom equation as well as their behavior. Behavior of integral curves in the neighborhoods of an arbitrary or integral curve are considered. The obtained results contain the answer to the question on approximation of solutions whose existence is established. The errors of the approximation are defined by functions that can be sufficiently small. The theory of qualitative analysis of differential equations and topological retraction method are used.
\end{abstract}

\section{INTRODUCTION}

Since introduced in the 1950s by P. A. Lagerstrom, the models of Lagerstrom equation were studied by many authors with the help of variational techniques (see [1] - [4]). Here we shall use the qualitative analysis theory and the topological retraction method ([5] - [11]).

The Lagerstrom equation is used in asymptotic treatment of viscous flow past a solid at low Reynolds number. In general form it is given by the non-autonomous second-order differential equation:

$$
y^{\prime \prime}+\left(\frac{n-1}{t}+y\right) y^{\prime}=0, \quad n \in \mathbb{N}, n \geq 1 .
$$

The cases $n=2$ and $n=3$ represent the physically relevant settings of flow in two and three dimensions, respectively.

We will consider the equation (1) on interval $I=(a, b)$, where $-\infty \leq$ $a<b<0$ or $0<a<b \leq+\infty$. Let

$$
\Gamma=\{(y, t) \in D: y=\psi(t), t \in I\},
$$

2010 Mathematics Subject Classification. 34C05.

Key words and phrases. The Lagerstrom equation, behavior of solutions, approximation of solutions. 
where $D=I_{y} \times I, I_{y} \subseteq \mathbb{R}$ and $\psi(t) \in C^{2}(I)$, be an arbitrary or integral curve of equation (1). We will establish some sufficient conditions on the existence and behavior of the classes of solutions of equation (1) in a certain region of the curve $\Gamma$, using the retraction method (method Wažewski).

Let $r_{1}, r_{2} \in C^{1}\left(I, \mathbb{R}^{+}\right)$and $\psi_{0}=\psi\left(t_{0}\right), \psi_{0}^{\prime}=\psi^{\prime}\left(t_{0}\right), y_{0}=y\left(t_{0}\right), y_{0}^{\prime}=$ $y^{\prime}\left(t_{0}\right), t_{0} \in I$. We shall consider the solutions $y(t)$ of equation (1) which satisfy on $I$, either one of the conditions

$$
\left|y_{0}-\psi_{0}\right|<r_{2}\left(t_{0}\right),\left|y_{0}^{\prime}-\psi_{0}^{\prime}\right|<r_{1}\left(t_{0}\right),
$$

or

$$
\frac{\left(y_{0}-\psi_{0}\right)^{2}}{r_{2}^{2}\left(t_{0}\right)}+\frac{\left(y_{0}^{\prime}-\psi_{0}^{\prime}\right)^{2}}{r_{1}^{2}\left(t_{0}\right)}<1
$$

Using substitution

$$
y^{\prime}=x,
$$

where $x=x(t)$ is a new unknown function, equation (1) is transformed into a quasilinear system of equations:

$$
\left.\begin{array}{l}
x^{\prime}=-\left(\frac{n-1}{t}+y\right) x \\
y^{\prime}=x \\
t^{\prime}=1
\end{array}\right\}
$$

defined on $\Omega=I_{x} \times D, I_{x} \subset \mathbb{R}$ the open interval, and curve $\Gamma$ is transformed into a curve $(\varphi(t), \psi(t), t), t \in I$, where $\varphi(t)=\psi^{\prime}(t)$.

We shall consider the behavior of the integral curves $(x(t), y(t), t)$ of the system (5) with respect to the sets $\sigma$ and $\omega$ :

$$
\sigma=\left\{(x, y, t) \in \Omega:|x-\varphi(t)|<r_{1}(t),|y-\psi(t)|<r_{2}(t)\right\}
$$

and

$$
\omega=\left\{(x, y, t) \in \Omega: \frac{(x-\varphi(t))^{2}}{r_{1}^{2}(t)}+\frac{(y-\psi(t))^{2}}{r_{2}^{2}(t)} \leq 1\right\} .
$$

The boundary surfaces of $\sigma$ and $\omega$ are, respectively:

$$
\begin{gathered}
X_{i}=\left\{(x, y, t) \in C l \sigma \cap \Omega: H_{i}^{1}(x, y, t)\right. \\
\left.:=(-1)^{i}(x-\varphi(t))-r_{1}(t)=0\right\}, i=1,2, \\
Y_{i}=\left\{(x, y, t) \in C l \sigma \cap \Omega: H_{i}^{2}(x, y, t)\right. \\
\left.:=(-1)^{i}(y-\psi(t))-r_{2}(t)=0\right\}, i=1,2, \\
W=\left\{(x, y, t) \in C l \omega \cap \Omega: H(x, y, t):=\frac{(x-\varphi(t))^{2}}{r_{1}^{2}(t)}+\frac{(y-\psi(t))^{2}}{r_{2}^{2}(t)}-1=0\right\},
\end{gathered}
$$


where $C l S,(S=\omega$ or $S=\sigma)$ is the set of all points of closure of $S$. ( $x$ is a point of closure of $S, S$ a subset of a Euclidean space, if every open ball centered at $x$ contains a point of $S$ (this point may be $x$ itself)).

Let us denote the tangent vector field to an integral curve $(x(t), y(t), t), t$ $\in I$, of the system (5) by $T$. The vectors $\nabla H_{i}^{1}, \nabla H_{i}^{2}$ and $\nabla H$ are the external normals on surfaces $X_{i}, Y_{i}$ and $W$, respectively. We have:

$$
\begin{gathered}
T(x, y, t)=\left(-\left(\frac{n-1}{t}+y\right) x, x, 1\right), \\
\nabla H_{i}^{1}(t)=\left((-1)^{i}, 0,(-1)^{i-1} \varphi^{\prime}-r_{1}^{\prime}\right), i=1,2, \\
\nabla H_{i}^{2}(t)=\left(0,(-1)^{i},(-1)^{i-1} \psi^{\prime}-r_{2}^{\prime}\right), i=1,2, \\
\frac{1}{2} \nabla H(x, y, t) \\
=\left(\frac{x-\varphi}{r_{1}^{2}}, \frac{y-\psi}{r_{2}^{2}},-\frac{(x-\varphi)^{2} r_{1}^{\prime}}{r_{1}^{3}}-\frac{(y-\psi)^{2} r_{2}^{\prime}}{r_{2}^{3}}-\frac{(x-\varphi) \varphi^{\prime}}{r_{1}^{2}}-\frac{(y-\psi) \psi^{\prime}}{r_{2}^{2}}\right) .
\end{gathered}
$$

By means of scalar products

$$
\begin{aligned}
& P_{i}^{1}(x, y, t)=\left(\nabla H_{i}^{1}, T\right) \text { on } X_{i}, \\
& P_{i}^{2}(x, y, t)=\left(\nabla H_{i}^{2}, T\right) \text { on } Y_{i},
\end{aligned}
$$

and

$$
P(x, y, t)=\left(\frac{1}{2} \nabla H, T\right) \text { on } W
$$

we shall establish the existence and behavior of integral curves of the system (5) with respect to the set $\sigma$ and $\omega$, respectively.

Let us denote with $S^{p}(I), p \in\{0,1,2\}$, class solutions $(x(t), y(t))$ of the system (5) defined on $I$ which depends of $p$ parameters. We will say that the class of solutions $S^{p}(I)$ belongs to a set $\eta(\eta=\omega$ or $\eta=\sigma)$ if the graphs of functions from $S^{p}(I)$ are contained in $\eta$. In such a case we write $S^{p}(I) \subset \eta$. For $p=0$ we have notation $S^{0}(I)$ which means that there is at least one solution $(x(t), y(t), t)$ on $I$ of system (5) whose graph lies in the set $\eta$.

The results of this paper are based on the following lemmas (see [6], [9]), which for the system (5) and sets $\sigma$ and $\omega$, have the form:

Lemma 1. If it is, for the system (5), the scalar product of $P(x, y, t)<0$ on $W\left(P_{i}^{k}(x, y, t)<0\right.$ on $\left.\gamma \sigma=X_{1} \cup X_{2} \cup Y_{1} \cup Y_{2}, i=1,2, k=1,2\right)$, then the system (5) has a class of solutions $S^{2}(I)$ which belongs to a set $\omega$, for every $t \in I$, i.e. $S^{2}(I) \subset \omega\left(S^{2}(I) \subset \sigma\right)$. 
Lemma 2. If it is, for the system (5), the scalar product of $P(x, y, t)>0$ on $W\left(P_{i}^{k}(x, y, t)>0\right.$ on $\left.\gamma \sigma=X_{1} \cup X_{2} \cup Y_{1} \cup Y_{2}, i=1,2, k=1,2\right)$, then the system (5) has a class of solutions $S^{0}(I)$ which belongs to a set $\omega$, for every $t \in I$, i.e. $S^{0}(I) \subset \omega\left(S^{0}(I) \subset \sigma\right)$.

Lemma 3. If it is, for the system (5), the scalar product of $P_{i}^{1}(x, y, t)<0$ on $X_{1} \cup X_{2}$ and $P_{i}^{2}(x, y, t)>0$ on $Y_{1} \cup Y_{2}$ (or vice versa), then the system (5) has a class of solutions $S^{1}(I)$ which belongs to a set $\sigma$ for every $t \in I$, i.e. $S^{1}(I) \subset \sigma$.

According to Lemma 1, the set $W\left(\gamma \sigma=X_{1} \cup X_{2} \cup Y_{1} \cup Y_{2}\right)$ is a set of points of strict entrance of integral curves of the system (5) with respect to the sets $\omega(\sigma)$ and $\Omega$. Hence, all solutions of system (5) which satisfy condition $\left|x_{0}-\varphi_{0}\right| \leq r_{1}\left(t_{0}\right),\left|y_{0}-\psi_{0}\right| \leq r_{2}\left(t_{0}\right),\left(x_{0}=x\left(t_{0}\right)\right)$ also satisfy conditions $|x(t)-\varphi(t)| \leq r_{1}(t),|y(t)-\psi(t)| \leq r_{2}(t)$ for every $t>t_{0}$, i.e. $S^{2}(I) \subset \omega\left(S^{2}(I) \subset \sigma\right)$.

According to Lemma 2, the set $W\left(\gamma \sigma=X_{1} \cup X_{2} \cup Y_{1} \cup Y_{2}\right)$ is a set of points of strict exit of integral curves of the system (5) with respect to the sets $\omega(\sigma)$ and $\Omega$. Hence, according to T. Wazewski's retraction method [11], system (5) has at least one solutions belonging to set $\omega(\sigma)$ for every $t \in I$, i.e. $S^{0}(I) \subset \omega\left(S^{0}(I) \subset \sigma\right)$.

According to Lemma 3 , the set $X_{1} \cup X_{2}$ is a set of points of strict entrance, and $Y_{1} \cup Y_{2}$ is a set of strict exit (or reversely) of integral curves of (5) with respect to the sets $\sigma$ and $\Omega$. According to the retraction method system (5) has a one-parameter-class of solutions belonging to set $\sigma$ for every $t \in I$, i.e. $S^{1}(I) \subset \sigma$.

\section{MAIN RESUlts}

Theorem 1. Let $\Gamma$ be an arbitrary curve and $r_{1}, r_{2} \in C^{1}\left(I, \mathbb{R}^{+}\right)$.

(a) If

$$
\begin{aligned}
\left|\left(\frac{n-1}{t}+\psi\right) \varphi+\varphi^{\prime}\right|< & \left(\frac{n-1}{t}+\psi\right) r_{1}+r_{1}^{\prime}-\left(|\varphi|+r_{1}\right) r_{2}, \\
& r_{1}(t)<r_{2}^{\prime}(t)
\end{aligned}
$$

on $\gamma \sigma=X_{1} \cup X_{2} \cup Y_{1} \cup Y_{2}$, then all solutions $y(t)$ of the problem (1), (2) satisfy the conditions

$$
|y(t)-\psi(t)|<r_{2}(t),\left|y^{\prime}(t)-\psi^{\prime}(t)\right|<r_{1}(t) \quad \text { for } t>t_{0} .
$$

(b) If

$$
\begin{aligned}
\left|\left(\frac{n-1}{t}+\psi\right) \varphi+\varphi^{\prime}\right|> & \left(\frac{n-1}{t}+\psi\right) r_{1}+r_{1}^{\prime}+\left(|\varphi|+r_{1}\right) r_{2}, \\
& r_{1}(t)<-r_{2}^{\prime}(t)
\end{aligned}
$$


on $\gamma \sigma=X_{1} \cup X_{2} \cup Y_{1} \cup Y_{2}$, then at least one solution to the problem (1), (2) satisfies the conditions (8).

(c) If the conditions (6) and (10) or (7) and (9) are satisfied, then the problem (1), (2) has a one-parameter class of solutions that satisfy the conditions (8).

Proof. We shall consider equation (1) through the equivalent system (5). Consider a system of integral curves of (5) respect to a set $\sigma$. For scalar products $P_{i}^{1}(x, y, t)$ on $X_{i}$ and $P_{i}^{2}(x, y, t)$ on $Y_{i}$ we have, respectively:

$$
\begin{aligned}
& P_{i}^{1}(x, y, t)=-(-1)^{i}\left(\frac{n-1}{t}+y\right) x+(-1)^{i-1} \varphi^{\prime}-r_{1}^{\prime} \\
&=-\left(\frac{n-1}{t}+\psi\right) r_{1}+(y-\psi) r_{1} \\
&+(-1)^{i}\left[-\left(\frac{n-1}{t}+\psi\right) \varphi+(y-\psi) \varphi-\varphi^{\prime}\right]-r_{1}^{\prime}, \\
& P_{i}^{2}(x, y, t)=(-1)^{i} x+(-1)^{i-1} \psi^{\prime}-r_{2}^{\prime}=(-1)^{i}(x-\varphi)-r_{2}^{\prime} .
\end{aligned}
$$

(a) According to the conditions (6) and (7), the following estimates are valid for $P_{i}^{1}(x, y, t)$ on $X_{i}$ and $P_{i}^{2}(x, y, t)$ on $Y_{i}$, respectively:

$$
\begin{gathered}
P_{i}^{1}(x, y, t) \leq-\left(\frac{n-1}{t}+\psi\right) r_{1}+r_{1} r_{2}+\left|\left(\frac{n-1}{t}+\psi\right) \varphi+\varphi^{\prime}\right|+|\varphi| r_{2}-r_{1}^{\prime}<0, \\
P_{i}^{2}(x, y, t) \leq r_{1}-r_{2}^{\prime}<0 .
\end{gathered}
$$

Accordingly, the set of $\gamma \sigma=X_{1} \cup X_{2} \cup Y_{1} \cup Y_{2}$ is set of points of strict entrance for the integral curves of the system (5) respect to sets $\sigma$ and $\Omega$. Thus, all solutions of the system (5) that satisfy the initial conditions

$$
\left|y_{0}-\psi_{0}\right| \leq r_{2}\left(t_{0}\right),\left|x_{0}-\varphi_{0}\right| \leq r_{1}\left(t_{0}\right),
$$

also satisfy the conditions

$$
|x(t)-\varphi(t)|<r_{1}(t),|y(t)-\psi(t)|<r_{2}(t) \text { for every } t>t_{0} .
$$

As the $y^{\prime}=x$ and $\varphi=\psi^{\prime}$, it is

$$
x_{0}-\varphi_{0}=y_{0}^{\prime}-\psi_{0}^{\prime}
$$

so, all the solutions of problems (1), (2) satisfies the conditions (8).

(b) Taking into account the conditions (9) and (10), the following estimates are valid for $P_{i}^{1}(x, y, t)$ on $X_{i}$ and $P_{i}^{2}(x, y, t)$ on $Y_{i}$, respectively:

$$
\begin{gathered}
P_{i}^{1}(x, y, t) \geq-\left(\frac{n-1}{t}+\psi\right) r_{1}+r_{1} r_{2}+\left|\left(\frac{n-1}{t}+\psi\right) \varphi+\varphi^{\prime}\right|-|\varphi| r_{2}-r_{1}^{\prime}>0, \\
P_{i}^{2}(x, y, t) \geq-r_{1}-r_{2}^{\prime}>0 .
\end{gathered}
$$


We conclude that the set $\gamma \sigma$ is set of points of strict exit integral curves of the system (5) with respect to the sets $\sigma$ and $\Omega$. Thus, according to the method of retraction T. Wažewski, system (5) has at least one solution in the set $\sigma$ for all $t \in I$. So the problem (1), (2) has at least one solution that satisfies the conditions (8).

(c) In this case, the set $\left(X_{1} \cup X_{2}\right) \backslash\left(Y_{1} \cup Y_{2}\right)$ is the set of points of strict entrance and the set $\left(Y_{1} \cup Y_{2}\right) \backslash\left(X_{1} \cup X_{2}\right)$ is the set of points of strict exit (or vice versa) integral curves of the system (5) with respect to the sets of $\sigma$ and $\Omega$. According to the retraction method system (5) has a one-parameter class of solutions in the set $\sigma$ for every $t \in I$. Thus, for the problem (1), (2), there is a one-parameter class of solutions that satisfy the conditions (8) .

In the special case, when $\Gamma$ is an integral curve of equation (1), from Theorem 1 it follows that:

Corollary 1. Let $\Gamma$ be an integral curve of (1) and $r_{1}, r_{2} \in C^{1}\left(I, R^{+}\right)$.

(a) If

$$
0<\left(\frac{n-1}{t}+\psi\right) r_{1}+r_{1}^{\prime}-\left(|\varphi|+r_{1}\right) r_{2}, \quad r_{1}(t)<r_{2}^{\prime}(t)
$$

on $\gamma \sigma=X_{1} \cup X_{2} \cup Y_{1} \cup Y_{2}$, then all solutions $y(t)$ of the problem (1), (2) satisfy the conditions

$$
|y(t)-\psi(t)|<r_{2}(t),\left|y^{\prime}(t)-\psi^{\prime}(t)\right|<r_{1}(t) \quad \text { for } t>t_{0} .
$$

(b) If

$$
0>\left(\frac{n-1}{t}+\psi\right) r_{1}+r_{1}^{\prime}+\left(|\varphi|+r_{1}\right) r_{2}, \quad r_{1}(t)<-r_{2}^{\prime}(t)
$$

on $\gamma \sigma=X_{1} \cup X_{2} \cup Y_{1} \cup Y_{2}$, then at least one solution to the problem (1), (2) satisfies the conditions (8).

(c) If the conditions (6) and (10) or (7) and (9) are satisfied, then the problem (1), (2) has a one-parameter class of solutions that satisfies the conditions (8).

Let us now consider solutions $y(t)$ of the equation (1) that satisfy condition (3), where $(\psi(t), t), t \in I$, is an arbitrary integral curve of the equation (1).

Theorem 2. Let functions $r_{1}, r_{2} \in C^{1}\left(I, \mathbb{R}^{+}\right)$and

$$
\left[(|\varphi|+|\psi|) r_{2}^{2}(t)+r_{1}^{2}(t)\right]^{2}<4 r_{1}(t) r_{2}(t)\left[\left(\frac{n-1}{t}+y\right) r_{1}(t)+r_{1}^{\prime}(t)\right] r_{2}^{\prime}(t) .
$$

Then: 
(i) If

$$
r_{2}^{\prime}(t)>0, t \in I
$$

then all solutions $y(t)$ of the problem (1), (3) satisfy the conditions

$$
\frac{(y-\psi(t))^{2}}{r_{2}^{2}(t)}+\frac{\left(y^{\prime}-\psi^{\prime}(t)\right)^{2}}{r_{1}^{2}(t)}<1, \text { for } t>t_{0} .
$$

(ii) If

$$
r_{2}^{\prime}(t)<0, t \in I
$$

then at least one solution to the problem (1), (3) satisfies the condition (13), where $y(t)=\psi(t)$ is the solution the equation (1).

Proof. We shall consider equation (1) through the equivalent system (5). Consider the integral curves of the system (5) with respect to a set of $\omega$ and $\Omega$. For the scalar product of $P(x, y, t)=\left(\frac{1}{2} \nabla H, T\right)$ on $W$, we have:

$$
\begin{aligned}
P(x, y, t) & =-\left(\frac{n-1}{t}+y\right) x \frac{x-\varphi}{r_{1}^{2}}+x \frac{y-\psi}{r_{2}^{2}}-\frac{(x-\varphi)^{2}}{r_{1}^{3}} r_{1}^{\prime} \\
& -\frac{(y-\psi)^{2}}{r_{2}^{3}} r_{2}^{\prime}-\frac{(x-\varphi) \varphi^{\prime}}{r_{1}^{2}}-\frac{(y-\psi) \psi^{\prime}}{r_{2}^{2}} \\
= & \left.-\left(\frac{n-1}{t}+y\right)(x-\varphi)-\left(\frac{n-1}{t}+y\right) \varphi\right] \frac{x-\varphi}{r_{1}^{2}}+\frac{(x-\varphi)(y-\psi)}{r_{2}^{2}} \\
& -\frac{(x-\varphi)^{2}}{r_{1}^{3}} r_{1}^{\prime}-\frac{(y-\psi)^{2}}{r_{2}^{3}} r_{2}^{\prime}-\frac{(x-\varphi) \varphi^{\prime}}{r_{1}^{2}}-\frac{(y-\psi) \psi^{\prime}}{r_{2}^{2}} .
\end{aligned}
$$

If we introduce the notation

$$
X=\frac{x-\varphi}{r_{1}}, Y=\frac{y-\psi}{r_{2}}
$$

we have:

$$
\begin{aligned}
P(x, y, t)= & {\left[-\left(\frac{n-1}{t}+y\right)-\frac{r_{1}^{\prime}}{r_{1}}\right] X^{2}+\frac{r_{1}}{r_{2}} X Y-\frac{r_{2}^{\prime}}{r_{2}} Y^{2} } \\
& +\left[-\left(\frac{n-1}{t}+y\right)-\varphi^{\prime}\right] \frac{X}{r_{1}} \\
= & {\left[-\left(\frac{n-1}{t}+y\right)-\frac{r_{1}^{\prime}}{r_{1}}\right] X^{2}+\frac{r_{1}}{r_{2}} X Y-\frac{r_{2}^{\prime}}{r_{2}} Y^{2} } \\
& +\left[\left(\frac{n-1}{t}+\psi\right)-\left(\frac{n-1}{t}+y\right)\right] \varphi \frac{X}{r_{1}} .
\end{aligned}
$$


The following estimates for $P(x, y, t)$ on the surface $W$ are valid:

$$
\begin{aligned}
P(x, y, t) \leq & {\left[-\left(\frac{n-1}{t}+y\right)-\frac{r_{1}^{\prime}}{r_{1}}\right] X^{2}+\left|\frac{r_{1}}{r_{2}}\right||X||Y|-\frac{r_{2}^{\prime}}{r_{2}} Y^{2} } \\
& +[|\varphi|+|\psi|] \frac{r_{2}}{r_{1}}|X||Y| \\
= & {\left[-\left(\frac{n-1}{t}+y\right)-\frac{r_{1}^{\prime}}{r_{1}}\right] X^{2} } \\
& +\left[(|\varphi|+|\psi|) \frac{r_{2}}{r_{1}}+\left|\frac{r_{1}}{r_{2}}\right|\right]|X||Y|+\left[-\frac{r_{2}^{\prime}}{r_{2}}\right] Y^{2}, \\
P(x, y, t) \geq & {\left[-p(y, t)-\frac{r_{1}^{\prime}}{r_{1}}\right] X^{2}-\left|-q(y, t) \frac{r_{2}}{r_{1}}+\frac{r_{1}}{r_{2}}\right||X||Y|+\left[-\frac{r_{2}^{\prime}}{r_{2}}\right] Y^{2} } \\
& -\left[L_{1}|\varphi|+L_{2}|\psi|+L_{3}\right] \frac{r_{2}}{r_{1}}|X||Y| \\
= & {\left[-p(y, t)-\frac{r_{1}^{\prime}}{r_{1}}\right] X^{2}-} \\
& -\left[\left(L_{1}|\varphi|+L_{2}|\psi|+L_{3}\right) \frac{r_{2}}{r_{1}}+\left|\frac{r_{1}}{r_{2}}-q(y, t) \frac{r_{2}}{r_{1} \mid}\right|\right]|X||Y| \\
= & {\left[-\frac{r_{2}^{\prime}}{r_{2}}\right] Y^{2} . }
\end{aligned}
$$

The right-hand sides of the above inequalities are the quadratic symmetric forms

$$
a_{11} X^{2} \pm 2 a_{12}|X||Y|+a_{22} Y^{2}
$$

where corresponding coefficients $a_{11}, a_{12}, a_{22}$ are introduced.

(i) Conditions (11) and (12) imply

$$
a_{22}<0, a_{11} a_{22}-a_{12}^{2}>0,
$$

which, according to Sylvester's criterion, means that $P(x, y, t)<0$ on $W$. Consequently, the set $W$ is the set of points of strict entrance to the integral curves of the system (5) respect to the sets $\omega$ and $\Omega$. Hence, all solutions of the system (5) that satisfy the initial condition

$$
\frac{\left(x_{0}-\varphi_{0}\right)^{2}}{r_{1}^{2}\left(t_{0}\right)}+\frac{\left(y_{0}-\psi_{0}\right)^{2}}{r_{2}^{2}\left(t_{0}\right)}<1
$$

satisfy the inequality

$$
\frac{(x(t)-\varphi(t))^{2}}{r_{1}^{2}(t)}+\frac{(y(t)-\psi(t))^{2}}{r_{2}^{2}(t)}<1, \text { for } t>t_{0} .
$$

Since $x_{0}-\varphi_{0}=y_{0}^{\prime}-\psi_{0}^{\prime}$, then all the solutions of problems (1), (3) satisfy condition (13). 
(ii) Conditions (11), (14) imply

$$
a_{22}>0, a_{11} a_{22}-a_{12}^{2}>0,
$$

which, according to Sylvester's criterion, means that $P(x, y, t)>0$ on $W$. Consequently, $W$ is the set of points of strict exit integral curves of the system (5) respect to the sets $\omega$ and $\Omega$. Hence, according to the retraction method, problem (5), (15) has at least one solution that satisfies the condition (16). Consequently, the problem (1), (3) has at least one solution that satisfies the condition (13).

Remark. We note that the obtained results also contain an answer to the question on approximation of solutions $y(t)$ whose existence is established. For example, the errors of the approximation for solutions $y(t)$ and derivative $y^{\prime}(t)$ in Theorem 1 are defined by the functions $r_{1}(t)$ and $r_{2}(t)$ which tend to zero as $t \rightarrow \infty$ and $r_{i}^{\prime}(t)<0,(i=1,2), t \in I$. For example, for the functions $r_{i}(t)$ we can use $r_{1}(t)=\alpha e^{-s t}$ and $r_{2}(t)=\beta e^{-p t}, s>0, p>0$ with parameters $\alpha$ and $\beta$, that can be arbitrarily small. In that case curve $\Gamma$ present a good approximation of solution $y(t)$ in $\sigma$.

\section{REFERENCES}

[1] D. S. Cohen, A. Fokas and P. A. Lagerstrom, Proof of some asymptotic results for a model equation for low Reynolds number flow, SIAM J. Appl. Math., 35 (1978), $187-207$.

[2] S. P. Hastings and J. B. McLeod, An elementary approach to a model problem of Lagerstrom, Siam J. Math Anal., 40 (6) 2009, 2421-2436.

[3] S. Rosenblat and J. Shepherd, On the asymptotic solution of the Lagerstrom model equation, SIAM J. Appl. Math., 29 (1975), 110-120.

[4] N. Popovic and P. Szmolyan, A geometric analysis of the Lagerstrom model problem, J. Differ. Equations, 199 (2004), 290-325.

[5] B. Vrdoljak, On solutions of the Lagerstrom equation, Arch. Math. (Brno), 24 (3) (1988), 111-222.

[6] A. Omerspahić, Retraction method in the qualitative analysis of the solutions of the quasilinear second order differential equation, Applied Mathematics and Computing (edited by M. Rogina et al.), Department of Mathematics, University of Zagreb, Zagreb, 2001, 165-173.

[7] B. Vrdoljak and A. Omerspahić, Qualitative analysis of some solutions of quasilinear system of differential equations, Applied Mathematics and Scietific Computing (edited by Drmač et al.), Kluwer Academic/Plenum Publishers, 2002, 323-332.

[8] B.Vrdoljak and A. Omerspahić, Existence and approximation of solutions of a system of differential equations of Volterra type, Math. Commun., 9 (2) (2004), 125-139.

[9] A. Omerspahić and B. Vrdoljak, On parameter classes of solutions for system of quasilinear differential equations, Proceeding of the Conference on Applied Mathematics and Scientific Computing, Springer, Dordreht, 2005, 263-272. 
[10] A. Omerspahić, Existence and behavior solutions of a system of quasilinear differential equations, Creat. Math. Inform., 17 (3) (2008), 487-492.

[11] T. Wažewski, Sur un principe topologique de l'examen de l'allure asymptotique des integrales des equations differentielles ordinaires, Ann. Soc. Polon. Math., 20 (1947), 279-313.

(Received: April 15, 2013)

(Revised: July 3, 2013)
Faculty of Mechanical Engineering

University of Sarajevo

Vilsonovo šetalište 9

71000 Sarajevo

Bosnia and Herzegovina

alma.omerspahic@mef.unsa.ba

hadziabdic@mef.unsa.ba 